\title{
Define and exploit guidelines for interactive redesign of products' User eXperience
}

\author{
Stefano Filippi ${ }^{1}\left[\right.$ B $\cdot$ Barbara Motyl ${ }^{1}$
}

Received: 10 March 2020 / Accepted: 23 September 2020 / Published online: 9 October 2020

(c) The Author(s) 2020

\begin{abstract}
Nowadays, User eXperience (UX) is one of the main concerns in developing innovative products. This research develops the way to define and exploit guidelines for interactive redesign of the UX of products. Because of their origin, these guidelines will encompass all UX characteristics reputed as meaningful like usefulness, subjective feelings, motivational aspects, emotions, etc.; moreover, the redesign suggestions offered by these guidelines, as well as the procedures to collect and analyse the data to define other guidelines and suggest the best ways to implement them, push different competencies (engineers, UX experts, psychologists, etc.) to work in synergy, just like an interactive design context requires.
\end{abstract}

Keywords Product User eXperience · Interactive UX redesign guidelines · Mental models · irMMs-based method · CUE model

\section{Introduction and background}

Because of its increasing importance in research and industrial contexts, the literature already offers many studies on User eXperience (UX) [1]. These studies propose several UX definitions, slightly different from each other [2]. Based on these definitions, the literature offers several UX design and evaluation methods and tools. Among them, the irMMsbased method, developed starting from the framework based on the product experience of Desmet and Hekkert, evaluates the quality of UX by exploiting interaction-related mental models (irMMs). In the last years, the irMMs-based method reached its release 2.0 thanks to the integration with the meCUE questionnaire [3]. The outcomes of this synergy suggested considering the irMMs-based method not only from the UX evaluation point of view but also for UX redesign [4]. This research received this suggestion and, starting from a deep investigation on the UX characteristics highlighted by the synergy, developed the way to define and exploit guidelines for interactive redesign of the UX of products starting

Stefano Filippi

filippi@uniud.it

Barbara Motyl

barbara.motyl@uniud.it

1 DPIA - University of Udine, Via delle Scienze 206, Udine, Italy from the analysis of real UX redesign case studies as well as from considering existing UX methods, principles, etc. Because of their origin, these guidelines will encompass all the UX facets reputed as meaningful in an interactive design context; moreover, they will be generic enough to be used in different contexts, on different products. Finally, the redesign suggestions offered by these guidelines will push different competencies (engineers, UX experts, psychologists, etc.) to work in synergy, just like an interactive design context requires [5]. The structure and functioning of the guidelines will be independent from the methods and tools to collect the data required to adopt them and this will widen their applicability even more. The guidelines should be useful for both researchers and practitioners. The former could deepen their knowledge about UX characteristics and about the relationships between characteristics and redesign processes, all of this resulting in improvements of existing product redesign methods and tools as well as in developing new ones following the same procedure adopted here. The latter could adopt the guidelines in improving their products even without specific knowledge about UX just because the suggestions are simple and use well-known methods and tools to be implemented. 


\section{Research methodology}

The research activities focus on the way to define and exploit UX redesign guidelines, as well as on a first adoption in the field. What follows describes all of this in detail.

\subsection{Definition of the UX redesign guidelines}

A UX redesign guideline consists of suggestions for redesign the UX of products; these suggestions will help researchers and practitioners in generating UX redesign solutions (solutions, hereafter) by implementing them case by case, due to the specific product to redesign. Different competencies generate the guidelines considering seven UX characteristics in order to cover the field exhaustively. These characteristics are: instrumental product qualities, non-instrumental product qualities, meanings, emotions, consequences of use, overall evaluation, differences between the expected user actions and product reactions/feedback and those allowed by the product under redesign. Guidelines can be generated by following the top-down and the bottom-up approaches. In the top-down approach, experts consider existing theories and guidelines and, starting from them, define new ones. Conversely, the bottom-up approach collects and analyses real-world data and uses them to define guidelines. The research described here suggests combining the two approaches. From the top-down side, good sources to refer to can be systematic reviews on UX design and evaluation [1, 6, 7], existing guidelines like the Nielsen's heuristics [8] and the Shneiderman's golden rules $[9,10]$, and design principles like those offered by the TRIZ theory [11, 12], like the set of sociotechnical principles to guide system design [13] and like the twelve principles for green engineering [14]. From the bottom-up side, this research suggests to consider real case studies of product UX redesign belonging to the academic and industrial fields. This combination occurs as follows. The results of the analysis of the case studies (bottom-up side) allows reasoning on the definition of the guidelines, while the collections of heuristics, rules and principles (top-down side) support in verifying the reasoning accuracy and in defining the guideline details. One of the most important aspects during the definition and exploitation of the guidelines regards data collection and classification, which must obey to rules aimed at making the selection of the suitable guidelines for the specific redesign context and product possible and effective. For what concern the format, each guideline will consist of the positive and the negative components. The positive component will contain suggestions on how to enhance aspects of the product UX already evaluated as positive. The negative component will suggest how to overcome and improve negative aspects of the product UX. The goals, present in both the components, will help researchers and practitioners in foreseeing the results that could be achieved thanks to

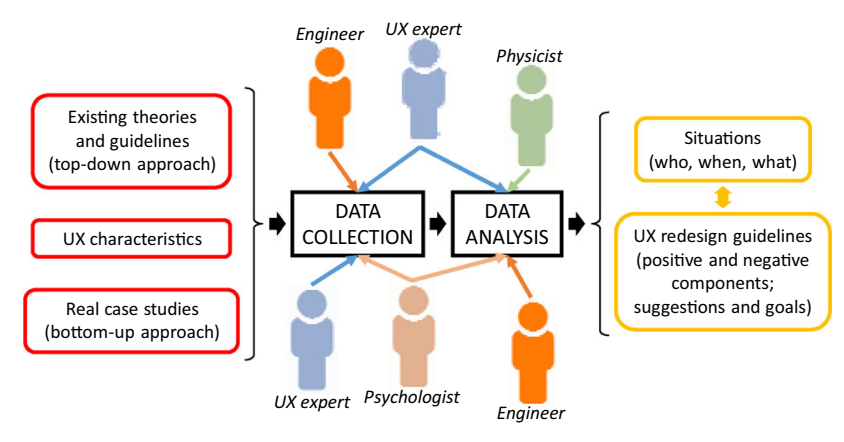

Fig. 1 Guidelines definition

the implementation of the suggestions. The considerations behind the definition of every guideline are collected all the way through. Their analysis and elaboration, obtained by deleting doubles, highlighting univocal references to guidelines, grouping against UX characteristics, etc., make them take the form of situations where UX strengths and flaws of the product to redesign show up. The suggestions of the guidelines simply aim at emphasizing those strengths and eliminating those flaws. All of this suggests identifying situations time by time, depending on the outcomes of the UX evaluation activities, and considering them like triggers of the most suitable guidelines to maximize the redesign effectiveness. Each situation considers a specific user role (who) that in a specific moment of interaction (when) highlights something referred to a specific UX characteristic (what). Each situation leads to a guideline. Figure 1 summarizes the guidelines definition.

\subsection{Development of the guidelines exploitation}

The exploitation of the guidelines occurs through three activities: data collection, guideline selection and guideline implementation into solutions. Data can be collected using almost any method of choice, provided that the rules cited before are obeyed. The selection of the guidelines occurs by checking the presence of the situations cited before in the collected data. Once selected, the suggestions of the guidelines are translated into solutions thanks to the hints about the competencies to involve and the methods and tools to adopt. Once this generation comes to the end, solutions are compared to each other to delete doubles. Then, solutions are classified against the interaction topics they refer to (specific procedures, product components, etc.) and, for each topic, they are ordered against the numbers of occurrences (computed before the deletion of the doubles). Figure 2 summarizes the guidelines exploitation. 


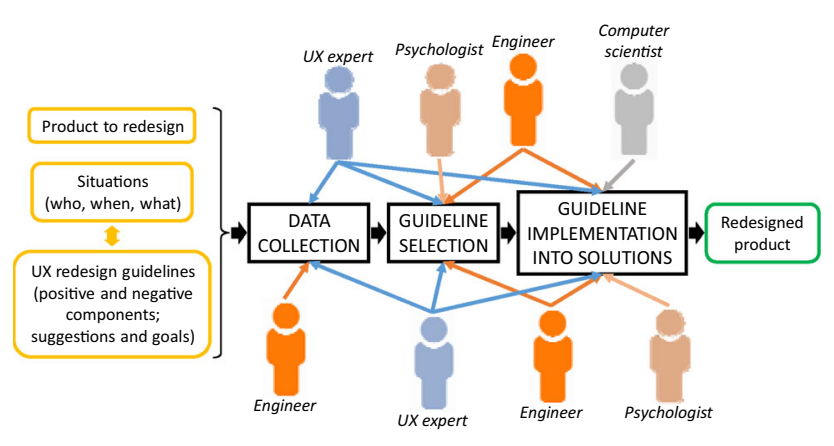

Fig. 2 Guidelines exploitation

\subsection{First adoption of the guidelines in the field}

The first adoption of the guidelines involves a state-of-the-art CAD software package developed by a well-known software house. Two UX experts and four practitioners-designers from the industry with basic knowledge about UX matters are involved. Data are collected using the irMMs-based method. Checking the situations list against the classified data selects four guidelines that are translated into interactive redesign solutions afterward.

\section{Results}

To date, the adoption of the way to define and exploit the UX interactive redesign guidelines generated ten guidelines and twenty-one situations. For example, referring to the eighth guideline, the suggestions of the positive component are "Make functionalities and working procedures highlighted as positive even more visible, accessible, effective and/or innovative. To do this, if these functionalities or working procedures refer to user actions, exploit usability/UX principles and guidelines like Nielsen's heuristics, Shneiderman's golden rules, etc.; on the contrary, if these functionalities or working procedures refer to product reactions/feedback, exploit rules and design principles like the TRIZ 40 principles, the twelve principles for green engineering, etc. Try to replicate all of this in all the other product functionalities and working procedures." and the goal is "Enhance product effectiveness in supporting user problem solving processes in all its functionalities.". The same guidelines offers as suggestions of the negative component "Improve negative actions/reactions/feedback by introducing suitable advices, alarms, helps and feedback by exploiting usability principles like the ten heuristics of Nielsen or the 40 principles of TRIZ." with the goal "Make the product able to drive users in performing their problem solving processes smoothly, easily and actively.". Two situations lead to this guideline. The first one consists of the absolute beginners user role (who) that, after the interaction (when), highlight something referred to the "user preferences between alternatives" of the UX characteristic "consequences of use" (what). The second one is the same, except for referring to the user role of relative beginners instead of that of the absolute ones.

\section{Conclusions}

The UX interactive design guidelines developed thanks to this research seem to be generic enough to be used in different situations and on different products. Each guideline consists of the goals it aims at and of the suggestions on what to do in order to further enhance positive product aspects or delete/correct negative ones. The involvement of different competencies in both the definition and exploitation of the guidelines Some research perspectives are as follows. The list of situations should be completed, with the natural repercussion on the need to develop further guidelines as well. To limit the heavy influence of researchers and practitioners' skill and knowledge on the generation of the solutions, the suggestions of the guidelines should be improved by detailing better each activity to do. A more objective assessment of the guidelines applicability and effectiveness requires more adoptions in the field.

Funding Open access funding provided by Università degli Studi di Udine within the CRUI-CARE Agreement.

Open Access This article is licensed under a Creative Commons Attribution 4.0 International License, which permits use, sharing, adaptation, distribution and reproduction in any medium or format, as long as you give appropriate credit to the original author(s) and the source, provide a link to the Creative Commons licence, and indicate if changes were made. The images or other third party material in this article are included in the article's Creative Commons licence, unless indicated otherwise in a credit line to the material. If material is not included in the article's Creative Commons licence and your intended use is not permitted by statutory regulation or exceeds the permitted use, you will need to obtain permission directly from the copyright holder. To view a copy of this licence, visit http://creativecomm ons.org/licenses/by/4.0/.

\section{References}

1. Zarour, M., Alharbi, M.: User experience aspects and dimensions: systematic literature review. Int. J. Knowl. Eng. 3(2), 52-59 (2017)

2. ISO 9241-210:2019.: Ergonomics of human-system interaction-Part 210: human-centred design for interactive systems (2019)

3. Filippi, S., Barattin, D.: Exploiting the meCUE questionnaire to enhance an existing UX evaluation method based on mental models. In: Marcus, A., Wang W. (eds.) Proceeding of the Interactional Conference of Human-Computer Interaction, Design, User Experience, and Usability. Practice and Case Studies, DUXU 2019, pp. 117-133. Springer, Orlando (2019)

4. Smith, S., Smith, G., Shen, Y.T.: Redesign for product innovation. Des. Stud. 33, 160-184 (2012) 
5. Briand, R., Fischer, X., Arrijuria, O., Terrasson, G.: Multidisciplinary design process based on virtual prototyping for microsystem design. Virtual Phys. Prototyp. 5, 153-162 (2010)

6. Quiñones, D., Rusu, C.: How to develop usability heuristics: a systematic literature review. Comput. Stand. Interfaces 53, 89-122 (2017)

7. Maia, C.L.B., Furtado, E.S.: A systematic review about user experience evaluation. In: Marcus A. (ed.) Proceeding of the International Conference on Human-Computer Interaction, DUXU 2016, Part I, LNCS 9746, pp. 445-455. Toronto, Canada (2016)

8. Nielsen, J., Molich, R.: Heuristic evaluation of user interfaces. In: Proceedings of the SIGCHI Conference on Human Factors in Computing Systems, CHI 90, pp. 249-256. Seattle, Washington, USA (1990)

9. Shneiderman, B., Plaisant, C.: Designing the User Interface: Strategies for Effective Human-Computer Interaction, 4th edn. Pearson Education Inc, New York (2005)

10. Aottiwerch, N., Kokaew, U.: Design computer-assisted learning in an online Augmented Reality environment based on Shneiderman's eight Golden Rules. In: Proceeding of the 14th International Joint Conference on Computer Science and Software Engineering, JCSSE 2017, Nakhon Si Thammarat, Thailand (2017)

11. Altshuller, G., Rodman, S.: The Innovation Algorithm: TRIZ, Systematic Innovation and Technical Creativity. Technical Innovation Center Inc (1999)

12. Cavallucci, D., Khomenko, N.: From TRIZ to OTSM-TRIZ: addressing complexity challenges in inventive design. Int. J. Product Dev. 4, 4-21 (2007)

13. Clegg, C.W.: Sociotechnical principles for system design. Appl. Ergon. 31, 463-477 (2000)
14. Anastas, P.T., Zimmerman, J.B.: Through the 12 principles of green engineering. Environ. Sci. Technol. A, 95-101 (2003)

Publisher's Note Springer Nature remains neutral with regard to jurisdictional claims in published maps and institutional affiliations. 